\title{
Automatic Segmentation of Veterinary Infrared Images with the Active Shape Approach
}

\author{
Tom Wirthgen ${ }^{1}$, Stephan Zipser ${ }^{1}$, Ulrike Franze ${ }^{2}$, Steffi Geidel ${ }^{2}$, \\ Franz Dietel ${ }^{3}$, and Theophile Alary ${ }^{4}$ \\ 1 Fraunhofer Institute for Transportation and Infrastructure Systems, \\ Zeunerstr. 38, 01069 Dresden, Germany \\ \{tom.wirthgen, stephan.zipser\}@ivi.fraunhofer.de \\ WWw.ivi.fraunhofer.de \\ 2 HTW Dresden, Pillnitzer Platz 2, 01326 Dresden, Germany \\ franze@htw-dresden.de, geidel@pillnitz.htw-dresden.de \\ www.htw-dresden.de \\ 3 HTWK Leipzig, Postfach 3011 66, 04251 Leipzig, Germany \\ dietel@ftz.htwk-leipzig.de \\ www.htwk-leipzig.de \\ 4 Universite de technologie de Troyes, France \\ theophile.alary@utt.fr \\ wWW .utt.fr
}

\begin{abstract}
Modern livestock farming follows a trend to higher automation and monitoring standards. Novel systems for a health monitoring of animals like dairy cows are under development. The application of infrared thermography (IRT) for medical diagnostics was suggested long ago, but the lack of suitable technical solutions still prevents an efficient use. Within the R\&D project VIONA new solutions are developed to provide veterinary IRT based diagnostic procedures with precise absolute temperature values of the animal surface. Amongst others this requires a reliable object detection and segmentation of the IR images. Due to the significant shape variation of interest objects advanced segmentation methods are necessary. The "active shape" approach introduced by Cootes and Taylor [7] is applied to veterinary IR images for the first time. The special features of the thermal infrared spectrum require a comprehensive adaptation of this approach. The modified algorithm and first results of the successful application on approximately two million IR images of dairy cows are presented.
\end{abstract}

Keywords: active shape segmentation, infrared imaging, precise temperature measurements, veterinary diagnostics.

\section{Motivation and State of the Art}

The trend to higher automation and monitoring standards in modern livestock production as well as more restrictive legal requirements for animal welfare support the development of novel systems for an automatic health monitoring of

A. Heyden and F. Kahl (Eds.): SCIA 2011, LNCS 6688, pp. 435 446, 2011.

(C) Springer-Verlag Berlin Heidelberg 2011 
livestock [4]. For the health monitoring of animals, like the dairy cows discussed here, infrared thermography (IRT) can be used. The use of IRT for medical diagnostic has already been suggested in 1956 [13 by Lawson. But in spite of many investigations IRT is still rarely applied in veterinary.

Actually the value of veterinary IRT is discussed controversy. A short application orientated survey gives Knizkova [12. In case of the IRT diagnostic of dairy cows Barth [1] concludes that IRT is not suitable for the early detection of subclinical udder infections. But Colak et al. 6] showed that all kinds of udder infections (clinical as well as subclinical) can be recognised with IRT. Similar results found Berry et al. 3]. In contrast to human medicine, were measurement and diagnostic standards where established in the last years [916, the present veterinary IRT still suffer from following disadvantages:

1. The lack of technical defined standards (required thermal resolution, maximal measurement uncertainty etc.) and procedural standards (camera pose, ambient conditions etc.) for the IRT measurement.

2. The elaborate determination of temperature values. The infrared (IR) image analysis is often performed on basis of a computer aided calculation of temperature values of regions of interest (ROI), e.g. the average temperature of udder quarters. Therefore first the two ROI left and right udder quarter must be localised and marked in the IR image. This take place by a faultprone and time consuming manual segmentation. As a consequence only a few IR images per animals can be analysed.

3. The diagnosis, i.e. the veterinary interpretation of the temperature values, strongly depends on the individual experience and cognitive skills.

Therefore the interdisciplinary $R \& D$ project VIONA was established whereat scientific and industrial partners are developing and evaluating a novel system for an automatic infrared based health monitoring. The focus is on monitoring

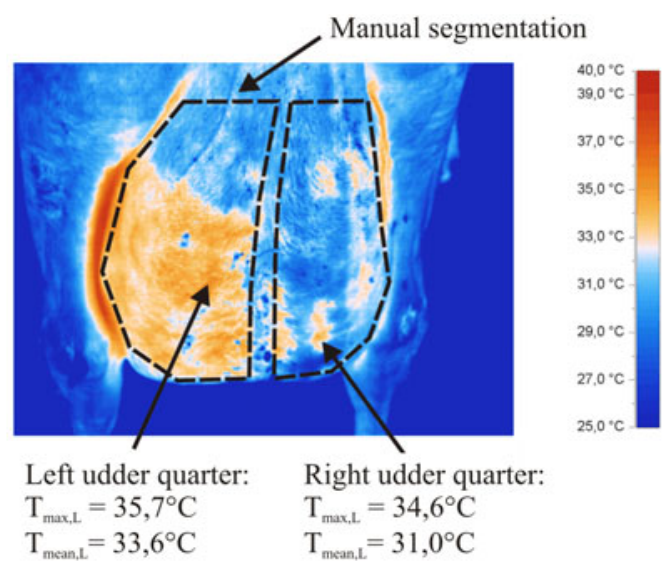

Fig. 1. Manual segmentation and feature extraction of an IR image of an udder 
of dairy cows under typical farming conditions like moving animals as well as varying ambient conditions. The paper discusses the objective precise IRT based temperature measurement. The main focus is the automatic extraction of precise temperature values, the basis of the veterinary diagnostic algorithms, Fig. 1 .

\section{Image Acquisition and IR Temperature Calculation}

Within the VIONA project many IRT measurements are realised with the configuration shown in Fig. 2. Two IR cameras 1 are used in combination with a reference body of known temperature and emissivity. This generates referenciated IR images sequences from dairy cows on a milking carousel. The ambient conditions are recorded and an identification refers the IR images to the individual animal. In the following the images from the rear IR camera are considered.

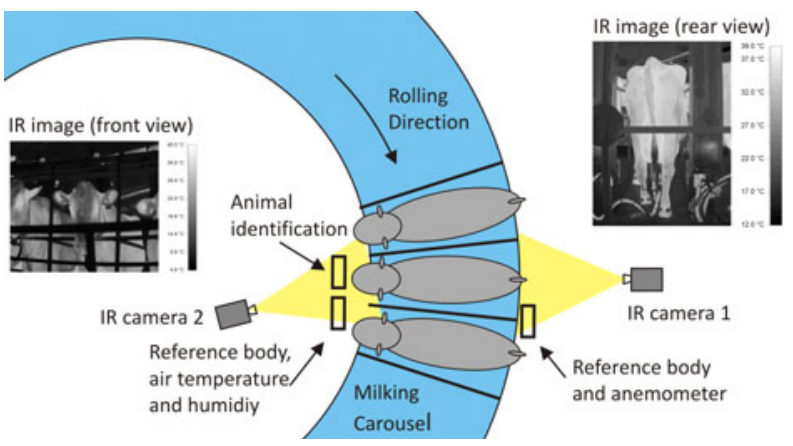

Fig. 2. IRT measurement of dairy cows on a milking carousel

Veterinary diagnostics require a "precise" temperature determination. Relevant are temperature differences of about $0.3 \mathrm{~K}, 15$. From the measurement point of view this implies first that the IRT measurement has to provide IR images with a low temperature uncertainty. Second an accurate image segmentation is necessary to calculate the diagnostic temperature values based on the "correct" ROI. Obviously both process steps influence the resulting temperature "precision".

State of the art IR cameras provide a temperature resolution of about $0.1 \mathrm{~K}$. A common mistake is the assumption that this high resolution is also valid for comparative analysis between different IR images, e.g. time series of images. The measuring uncertainty is ignored in nearly all veterinaty studies. An analysis of temperature measurement uncertainty according to the procedure defined by the industry measurement standard DIN1319 [14] shows, that primary due to sensor drift the resulting temperature uncertainty lies at about $\pm 2.2 \mathrm{~K}$ even if the

${ }^{1}$ DIAS Infrared: PYROVIEW 640L, 640×480 pixels, spectral range 8-14 $\mu \mathrm{m}$, measurement uncertainty $\pm 2.0 \mathrm{~K}$, temperature resolution $<0.1 \mathrm{~K}$. 

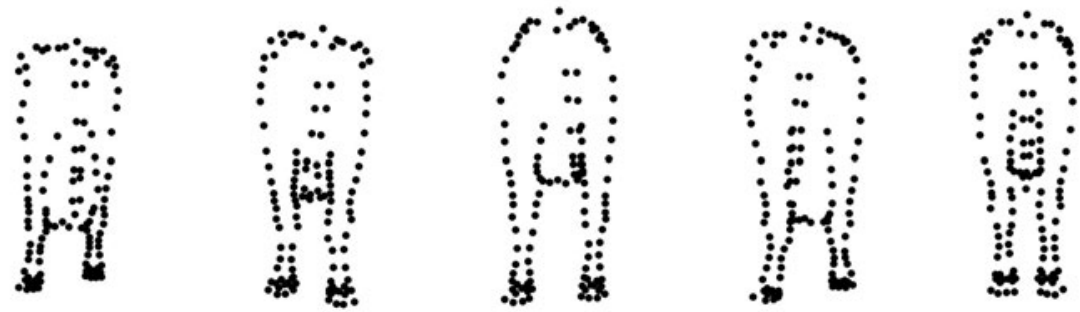

Fig. 3. Exemplary landmarks of "training shapes" for the dairy cow shape model

conventional radiant temperature correction [2] is performed. With the help of a model based temperature correction in combination with a reference bodies of exactly known emission properties leads to a temperature uncertainty of about $\pm 0.4 \mathrm{~K},[18$.

\section{IRT Segmentation and the Active Shape Approach}

To investigate the potential of segmentation approaches fixed shape model approaches were evaluated in former investigations [17. Within this investigation a set of models could be found that provide a satisfying segmentation compared to the results of an manual segmentation. The used image resolution of $320 \times 240$ pixels shows some disadvantages for the feature extraction due to the fact that some anatomic structures are very small. Therefore a high resolution IR camera of $640 \times 480$ pixels (DIAS Infrared: PYROVIEW 640L) is used for further investigations. As a consequence the shape and pattern are depicted much more detailed. This variety could not be governed by a fixed model approach.

Based on the surveys of model based matching algorithms 511] a promising approach was chosen - the Active-Shape-Models (ASM), introduced by Cootes and Taylor $[8$. This approach involves three separated parts that can be optimized individually:

1. The shape representation as a parametric statistical model.

2. The statistical grey value model for the landmarks 2 .

3. The search algorithm using both models.

The shape is represented by a vector $\boldsymbol{x}=\left(x_{1}, y_{1}, \ldots, x_{m}, y_{m}\right)^{T}$ including the $m$ landmarks of the shape (Fig. 3). Based on a manually labelled set of "training shapes" the shape model is statistically derived. The parametric model consisting of the mean shape $\overline{\boldsymbol{x}}$ and a deviation term:

$$
\boldsymbol{x}=\overline{\boldsymbol{x}}+P \boldsymbol{b} .
$$

The dimension of the deviation term is reduced by a principle component analysis (PCA) and leads to the compact deviation term $P$. The limitation of the

${ }^{2}$ Landmarks are characteristic points of the object, in most cases they are equally spaced along borders and at junctions. 

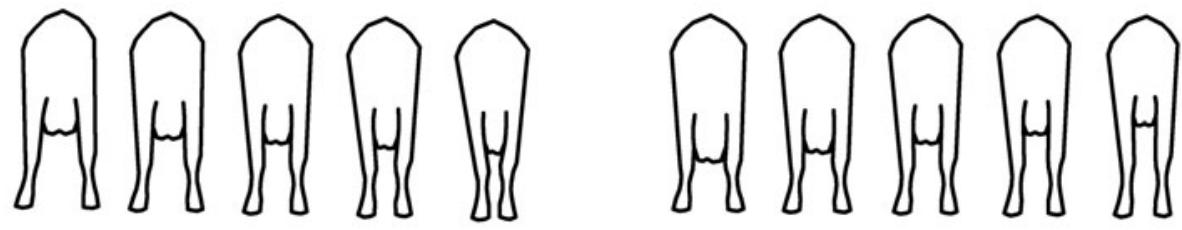

Fig. 4. Visualisation of the modelled shape variation $\boldsymbol{x}(\boldsymbol{b})$ left: parameter $b_{1}=\{-2 ;-1 ; 0 ; 1 ; 2\}$ - right: parameter $b_{2}=\{-2 ;-1 ; 0 ; 1 ; 2\}$

parameters $\boldsymbol{b}$ ensure that the model represents valid shapes and allows the coverage of shapes not included in the training set. Fig. 4 shows exemplary the shape variation due to the first two components (modes) of the model parameter vector $\boldsymbol{b}$. The parameters in general effect the whole shape, but some mainly effect specific characteristic, e.g. the second parameter $b_{2}$ mainly influence the height of the udder.

The grey value model describes the appearance of each landmark (LM). The model uses a normalised grey value profile $\boldsymbol{g}_{\boldsymbol{n}}$ in a line-shaped surrounding $j$ of a LM, giving the grey value vector $\boldsymbol{g}$ for each landmark:

$$
\boldsymbol{g}_{\boldsymbol{n}}=\frac{1}{\sum_{(j)} g_{j}} \boldsymbol{g} .
$$

Using this normalized grey value profile for each image in the training set the mean profile $\overline{\boldsymbol{g}}_{n}$ and the covariance matrix $C_{g}$ are generated for each LM.

Finally both models are joined by a search algorithm performing an iterative fitting process repeating the following steps:

1. Projecting the shape in the image.

2. Search best fitting landmarks in the shape points environment, by minimising the weighted distance between the model $\overline{\boldsymbol{g}}_{n}$ and the image grey $\boldsymbol{g}_{n, i}$

$$
D^{2}=\left(\boldsymbol{g}_{n, i}-\overline{\boldsymbol{g}}_{n}\right)^{T} C_{g}\left(\boldsymbol{g}_{n, i}-\overline{\boldsymbol{g}}_{n}\right) .
$$

3. Calculate the shape parameters using the fitted landmarks.

4. Normalisation of the shape by parameter limitation.

The use of ASM approach according to the configuration of 7] optimised for image of visible spectal range leads to a poor segmentation quality in case of veterinary IR images. An analysis shows following reasons:

- The image contrast is dominated by the ambient temperature, see Fig. 5 This makes normalised derivative landmarks inadequate.

- Parts of the shape model have great variance in shape or align and lead to undesirable feedback to other body parts in the model.

- Textured objects show unspecific grey value profiles due to the influence of view angle, environmental conditions, coat structure or dirt, see Fig. 5 .

To achieve improved segmentation results the known ASM approach was adapted to the specific features of images from the thermal infrared spectrum. 

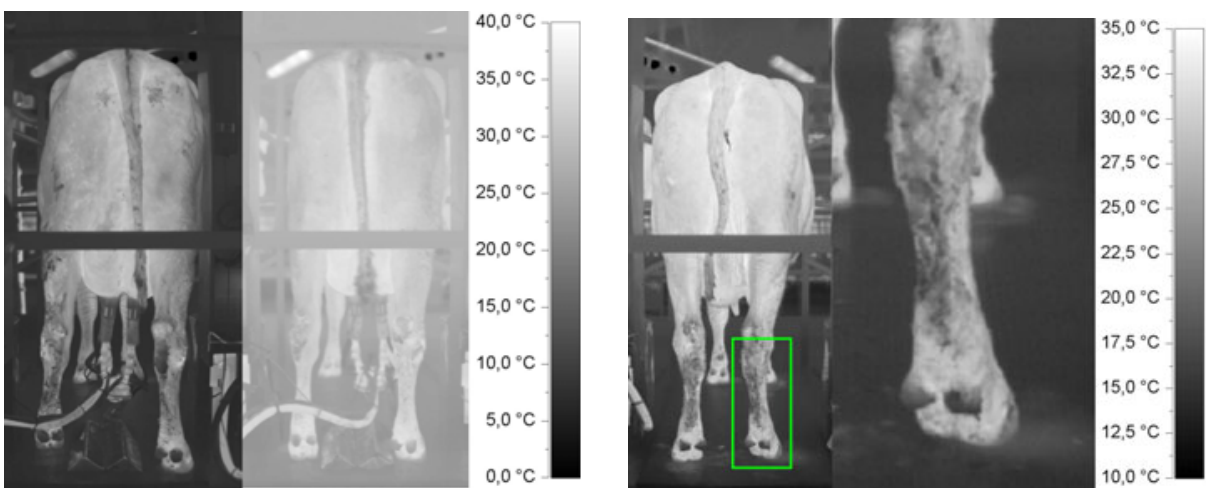

Fig. 5. Properties of IR images- left: influence of the ambient temperature (left: $9{ }^{\circ} \mathrm{C}$, right: $27^{\circ} \mathrm{C}$ ) to IR image contrast - right: texture disturbance due to coat

\section{Choice of Segmentation Criterion}

For the evaluation of the segmentation algorithm an objective criterion is necessary. The common mean point displacement 8] (for the $m$ landmarks of the fitted shapes $\boldsymbol{x}_{f}$ against the training shapes $\boldsymbol{x}_{t}$ over $n$ images):

$$
\delta=\frac{1}{m n} \sum_{i=1}^{n} \sqrt{\left(\boldsymbol{x}_{f, i}-\boldsymbol{x}_{t, i}\right)^{T}\left(\boldsymbol{x}_{f, i}-\boldsymbol{x}_{t, i}\right)}
$$

is not suitable as segmentation quality criterion. A point displacement is not always leads to a change in segmentation (see Fig. 6). Therefore a different criterion describing the enclosed area calculated with the segmentation area of the training set $a_{t}$ and the fitted model $a_{f}$ is suggested:

$$
A=\frac{\bigcap\left(a_{f}, a_{t}\right)}{\bigcup\left(a_{f}, a_{t}\right)} .
$$

This criterion is sensitive to segmentation differences and insensitive to point displacements along the segmentation borders, Fig. 6 .
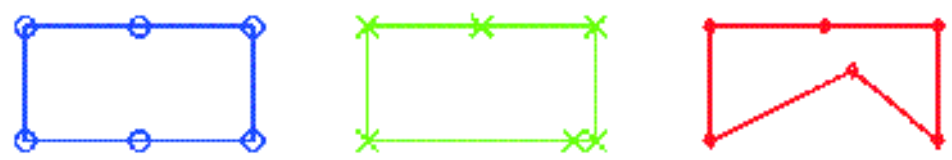

Fig. 6. Evaluation of the Segmentation: training shape (left), "correct" segmentation (mid, criterion $A=1,0$ ), and "incorrect" segmentation (right, criterion $A=0,69$ ) 
For applications the finding rate $R_{\alpha}$

$$
R_{\alpha}=\frac{1}{n} \sum S(A, \alpha) \quad \text { where } S(A, \alpha)= \begin{cases}1, & A>\alpha \\ 0, & \text { otherwise }\end{cases}
$$

is calculated. The finding rate $R_{\alpha}$ describes, how many "correct" segmentations were found for a set of $n$ test images.

\section{Specific Modifications of the ASM Segmentation}

Following steps of the ASM approach were modified for IR image segmentation:

1. Image preprocessing,

2. Landmark representation,

3. Landmark selection for shape creation and initialisation.

\subsection{Image Preprocessing}

The first modification addresses the disturbing effect of the ambient temperature on the image contrast. To "normalise" the IR images following preprocessing approaches were evaluated:

- Scaling to ambient temperature based interval (SAT),

- SAT and edge filter (Canny, Sobel, gradient),

- SAT and smoothing filter (mean, gauss and median), and

- Local histogram equalisation (LHE) [10].

Where the first three options gives negligible improvements the LHE shows good results, Fig. 17. The ambient temperature influence is reduced significantly and the landmark representations are much more stable. Even if the influence is not fully compensated, the grey value normalization Equ. (2) becomes obsolete.

\subsection{Landmark Representation}

As mentioned above, especially for body parts with coat the images show unspecific grey value profiles for the landmarks, see Fig. 5 The disturbing effect can be reduced by applying e.g. median filters. More efficient is the use of two dimensional temperature patterns instead of the conventional profile lines for landmark description.Investigation showed that 2D patterns have a generalising effect over textured landmark surroundings.

\subsection{Shape Creation and Initialisation}

During the investigation of the ASM approach it was noticed that certain body parts (e.g. the legs and the tail) show a significant different scale of variation. This is critical for the creation of proper shape models because within the model 


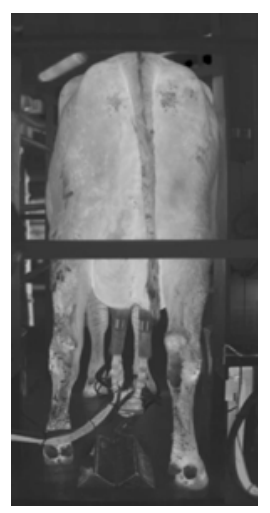

Orig. image A

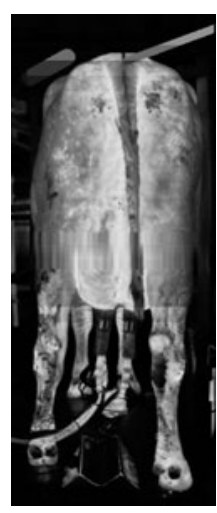

Prefilt. image A

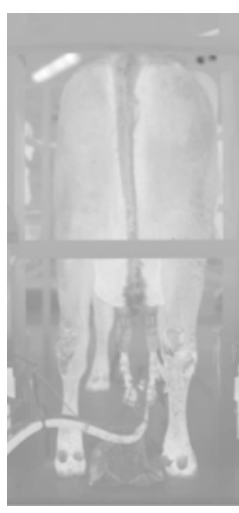

Orig. image B

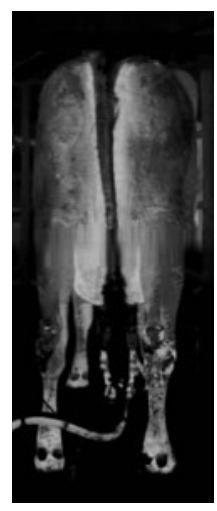

Prefilt. image B

Fig. 7. Preprocessing for IR images of different ambient temperatures; Remark: the horizontal bar is removed by interpolation; image $A: 9^{\circ} \mathrm{C}$, image $B: 27^{\circ} \mathrm{C}$

reduction step, the PCA "prefers "great form variations. This implies unintended couplings and the rejection of small varying body parts.

A possibility to handle this is the use of non equidistant aligned landmarks to equal the body parts weight in the model. In combination with the exclusion of body parts with high variance (e.g. the tail, which appears to be of no diagnostic interest) this algorithm shows good segmentation results.

The shape initialisation has a great impact on convergence and segmentation quality due to the fact that the ASM is a local search algorithm. For this reason an adapted pre-fitting for the shape was developed. Table 1 shows that the segmentation with the adapted initialisation is better than a segmentation using more pyramidal levels (which is equivalent to a great search space) and leads with $A>0.7$ for the claw and $A>0.8$ for the udder to a high segmentation quality.

All these modifications make a parameter set optimisation for the ASM search algorithm necessary. Therefore parameters as the size and alignment of the search area profile, the size of the grey value model pattern and the method of shape model parameter limitation were examined and adopted. The results of this optimisation are not discussed in detail, but the landmark pattern size optimisation will be exemplified. The criteria introduced above are calculated for different

Table 1. Influence of the initialisation and different number of pyramid levels, basis is a test set of 101 images and a shape model of 68 landmarks

\begin{tabular}{ccccc}
\hline Adapted & Pyramidal & \multicolumn{3}{c}{ Segmentation Criterion } \\
\cline { 3 - 5 } Initialisation & Levels & $A$ "Udder" & $A$ "Right claw" & $A$ "Left claw" \\
\hline no & {$\left[\frac{1}{2} ; 1\right]$} & 0.68 & 0.41 & 0.37 \\
no & {$\left[\frac{1}{4} ; \frac{1}{2} ; 1\right]$} & 0.74 & 0.71 & 0.58 \\
yes & {$\left[\frac{1}{2} ; 1\right]$} & 0.84 & 0.76 & 0.71 \\
\hline
\end{tabular}




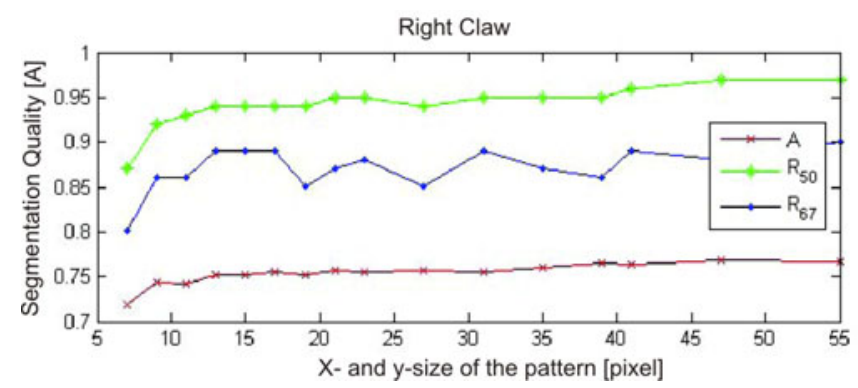

Fig. 8. Influence of landmark pattern size on segmentation quality

pattern sizes and test regions. Fig. 8 shows the results for the ROI "right claw". Summarised larger patterns show better segmentation quality, but require higher computational power.

\section{Application Results}

The ASM was successfully applied for the segmentation of more than two million IR images. The shape model was used as a framework to define veterinary ROI for objects like the right and the left quarter of the udder automatically. Based on the determined ROI for every IR image about 30 IR features, especially temperature values, were calculated for the development of the veterinary diagnostic approaches.

Table 2. Correlation coefficients between the IR features calculated from manually and automatically generated ROI

\begin{tabular}{lccccc}
\hline IR feature & $\begin{array}{c}\text { claw ROI } \\
\text { variant } 1\end{array}$ & $\begin{array}{c}\text { claw ROI } \\
\text { variant } 2\end{array}$ & $\begin{array}{c}\text { claw ROI } \\
\text { variant } 3\end{array}$ & udder & rear section \\
\hline$T_{\text {mean }}$ & 0.84 & 0.82 & 0.85 & 0.66 & 0.93 \\
$T_{\text {max }}$ & 0.89 & 0.88 & 0.88 & 0.76 & 0.85 \\
& & $\ldots$ & & \\
\hline
\end{tabular}

For the evaluation of the automatic image analyse a manually labelled test set of about 11000 images each with 7 ROI was created. The automatically created IR features show a high correlation to those calculated from manually segmented images (Tab. 2). Fig. 9 confirms this showing four time series of a cow over 34 days. The IR features $T_{\text {mean }}$ as well as the $T_{\text {max }}$ are nearly equal for manually and automatically generated ROI.

For the modified ASM approach in most cases a fast convergence is observed, typical are less then 15 iterations. The Fig 10 depicts the first four steps of a fitting process. The sidewards shift in an image sequence due to the milking 

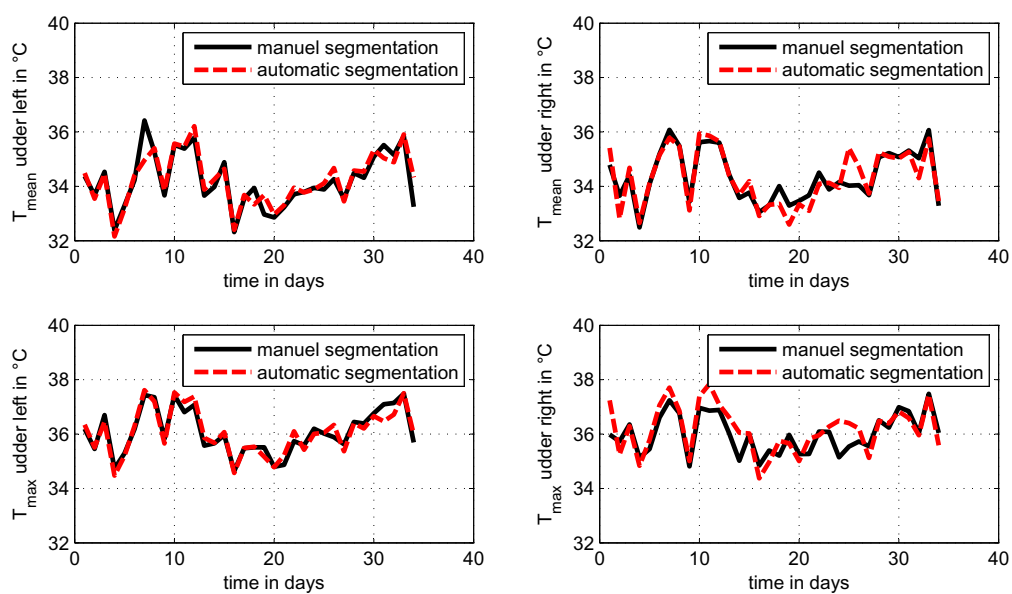

Fig. 9. IR feature $T_{\text {mean }}$ and $T_{\text {max }}$ time series for the ROI "udder left" and "udder right" of one cow (comparison of manually and automatically created ROI)

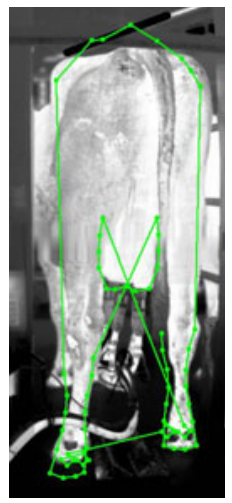

Step 1

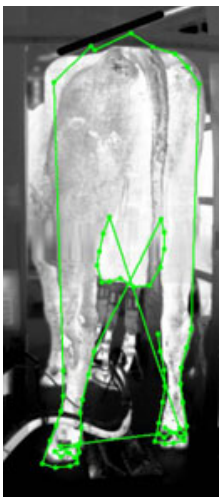

Step 2

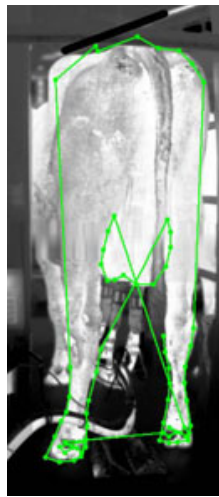

Step 3

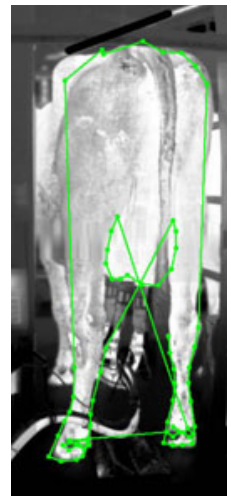

Step 4

Fig. 10. Fitting progress for an IR image

carousel movement refits the algorithm quickly. The main drawbacks for the veterinary IRT application have been solved by an adoption of the ASM algorithm.

The modification of the ASM approach for veterinary IR images could be summarised as follows:

- The specific features of IR images require an adapted preprocessing, best results were found for a combination of normalisation on ambient temperature (SAT) and local histogram equalisation (LHE).

- For the landmark model "large" 2D-patterns are suggested, which are much more robust than line profiles. 
- The suggested segmentation criterion is appropriate for numerical evaluation and also conform to a manual review.

The finding rate $R_{\alpha}$ could be increased from up to $60 \%$ for the fix model approach to more than $80 \%$ for the ASM segmentation. The image processing is implemented in the HALCON software and runs on standard computer hardware. The current processing capacity is approximately three images per second.

\section{Conclusions and Future Work}

The evaluation of the potential of veterinary IRT requires a precise IR temperature features based on a reliable automatic image segmentation. Due to the significant varying object shapes an advanced ASM approach was chosen and adapted to the specific properties of thermal IR images.

The approach was successfully tested on two million IR images. Further investigations face the development of a benchmark for the segmentation and runtime optimisation as well as an adaption to more distinct animal movements, maybe even "walking" animals. Moreover suitable segmentations criteria without a ground truth to detect incorrect segmentations (Fig. 11) has to be developed.

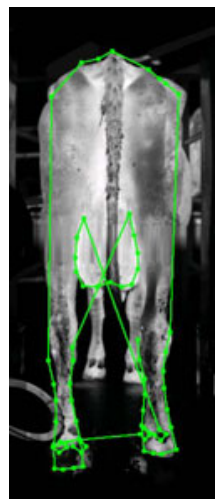

Missed left claw

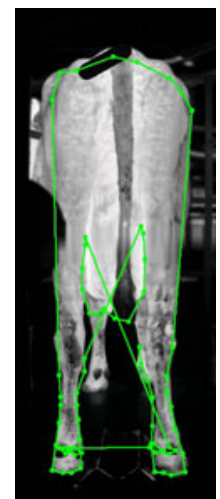

Missed udder

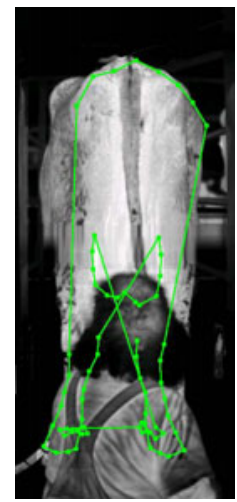

Occluding person

Fig. 11. Examples for missed shape model fits

\section{Acknowledgement}

This investigations are done within the scope of the interdisciplinary VIONA project (www.viona-system.net) financed by the German Federal Ministry of Education and Research (BMBF, ID 03WKP04B). The authors are grateful to the industry partners of the project DIAS Infrared, Ralle Landmaschinen, and Yoo as well as the hosting farm Methauer Agro AG. 


\section{References}

1. Barth, K.: Basic investigations to evaluate a highly sensitive infraredthermographtechnique to detect udder inflammation in cows. Milchwissenschaft 55(4), 607-609 (2000)

2. Bernhard, F.: Technische Temperaturmessung. In: VDI-Buch. Springer, Berlin (2004)

3. Berry, R., Kennedy, A., et al.: Daily variation in the udder surface temperature of dairy cows measured by infrared thermography: Potential for mastitis detection. Can. Journal of Animal Science 83(4), 687-693 (2003)

4. Büscher, W.: Current developments in livestock farming technology. Yearbook Agricultural Engineering 2011 23, 7-15 (2011)

5. Cheung, K.W., Yeung, D.Y., Chin, R.T.: On deformable models for visual pattern recognition. Pattern Rec. 35(7), 1507-1526 (2002)

6. Colak, A., Polat, B., et al.: Short Communication: Early Detection of Mastitis Using Infrared Thermography in Dairy Cows. J. Dairy Sci. 91(4), 4244-4248 (2008)

7. Cootes, T.: Model-Based Methods in Analysis of Biomedical Images. In: Image Processing and Analysis, pp. 223-248. Oxford University Press, Oxford (2000)

8. Cootes, T., Taylor, C.: Active shape model search using local grey-level models: A quantitative evaluation. In: Illingworth, J. (ed.) British Machine Vision Conference, pp. 639-648. BMVA Press (1993)

9. Diakides, N., Bronzino, J.: Medical infrared imaging. CRC Press, Boca Raton (2008)

10. Jähne, B.: Digital Image Processing. Springer, Weinheim (1997)

11. Jain, A.K., Zhong, Y., Dubuisson-Jolly, M.P.: Deformable template models: A review. Signal Processing 71(2), 109-129 (1998)

12. Knizkova, I., Kung, P.: Applications of infrared thermography in animal production. J. of Agric. Faculty of Ondokuz Mayis University 22(3), 329-336 (2007)

13. Lawson, R.: Implications of surface temperatures in the diagnosis of breast cancer. Canadian Medical Association Journal 75(4), 309 (1956)

14. Fundamentals of metrology: evaluation of measurements; uncertainty of measurement. DIN1319-4. Beuth Verlag, berlin (February 1999)

15. Clinical thermometers - part 5: Performance of infra-red ear thermometers (with maximum device). DIN EN 12470-5. Beuth Verlag, berlin (September 2003)

16. Ring, E.F.J., McEvoy, H., et al.: New standards for devices used for the measurement of human body temperature. J. Med. Eng. Technol. 34(4), 249-253 (2010)

17. Wirthgen, T.: Entwicklung und Berechnung IR-basierter Kenngrößen zur Analyse des Gesundheitszustandes von Kühen. diplom thesis, TU Dresden (2007)

18. Wirthgen, T., Zipser, S., et al.: Precise ir-based temperature measuring - a case study for the automatic health monitoring of dairy cows. In: SENSOR+TEST Conference - IRS ${ }^{2},(2011)$ (accepted paper) 